\title{
Very Short Gamma Ray Bursts Study and Primordial Black Holes
}

\section{David B. Cline}

UCLA Dept. Of Physics \& Astronomy

P.O. Box 951457, Los Angeles CA 90095 USA

E-mail: dcline@physics.ucla.edu

\section{Stanislas Otwinowski}

UCLA Dept. Of Physics \& Astronomy

CERN, CH-1211, Geneve 23, Switzerland

E-mail: stanislaw.otwinowskidcern. ch

We present the state of current research of Very Short Gamma Ray Bursts (VSGRBs) from BATSE, KONUS and SWIFT detectors. We found that VSGRBs form a distinguished class of GRBs, which in our opinion in most cases is composed of the primordial black hole evaporation events. Arguments supporting our opinion: (a) Time duration $\left(\mathrm{T}_{90}\right)$ distribution of VSGRBs for BATSE, KONUS and SWIFT detectors [2]; (b) Visible anisotropy in the galactic angular distribution of BATSE VSGRB events; (c) V/ Vmax distribution for BATSE VSGRB events indicating the local distance production [3]; (d) Practically no afterglows in SWIFT VSGRB sample $(25 \%)$, in contrast to the noticeable frequency in SGRB sample (78\%); (e) Time profile of rising part BATSE VSGRBs in agreement with evaporation PBH model. 
We divide GRB from detector BATSE into three classes according to their time duration $\left(\mathrm{T}_{90}\right)$ : long L ( $\left.\mathrm{T}_{90}>1 \mathrm{~s}\right)$; short $\mathrm{S}\left(1 \mathrm{~s}>\mathrm{T}_{90}>0.1 \mathrm{~s}\right)$; and very short VS $\left(\mathrm{T}_{90}<0.1 \mathrm{~s}\right),[1]$. We assume that the VSGRBs constitute a separate class of GRBs with log normal duration distribution and we fit the time distribution, with a three-population model. The fit is excellent but does not give significant evidence for a three-population model [1].

We have also analyzed SGRBs from the KONUS-WIND detector. Nominal energy range of gamma-ray measurements covers the interval from $12 \mathrm{keV}$ up to $10 \mathrm{MeV}$. This gives the possibility of comparing SGRBs ( $\mathrm{T}_{90}<2 \mathrm{~s}$ ) of the Low Energy Data (LED), $<\gamma><90 \mathrm{keV}$, with High Energy Data (HED), $<\gamma>>90 \mathrm{keV}$, Fig. 1. LED are normalized to HED in region $(0.0-$ $1.6 \mathrm{~s}$ ) and comparing both distribution we see strong disagreement (prob. $<10^{-10}$ ). We observe a large excess of VSGRBs with $\langle\gamma\rangle>90 \mathrm{keV}$, Fig. 1. Like BATSE KONUS uses the shortest trigger of $64 \mathrm{~ms}$, so we should observe even bigger excess because the detector lost detection efficiency at $\mathrm{T}_{90}<64 \mathrm{~ms}$, as in the BATSE case. This is an additional argument to treat VSGRBs as a separate class of events.

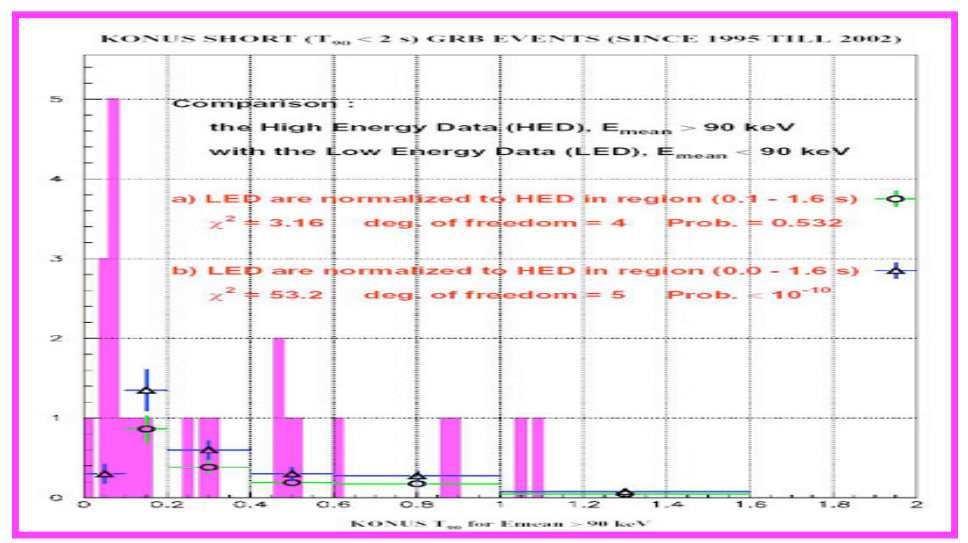

Fig. 1. KONUS data with different cuts on the average energy gamma, $<\gamma>[2]$.

Galactic angular distribution for BATSE events [3] for VSGRBs $\left(\mathrm{T}_{90}<0.1 \mathrm{~s}\right)$ are strongly grouped in $1 / 8$ of the whole space, giving an excess with probability 0.00007 to be a fluctuation from Poisson distribution. See also Fig. 3.

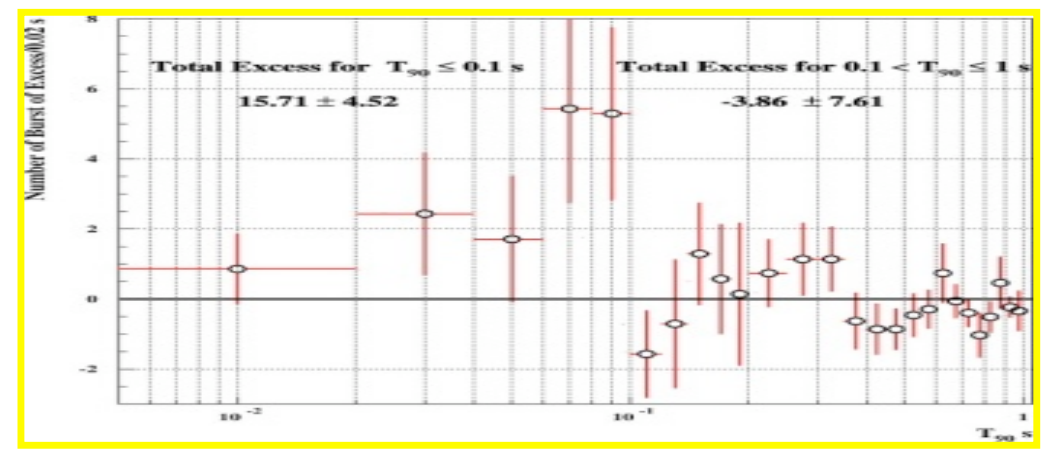

Fig. 2. BATSE GRB events (1991 Apr 21 - 2000 May 26). Excess in GRBs inside the chosen region (30 $\left.<\mathrm{b}<-30^{\circ} ; 90^{\circ}<1<180^{\circ}\right)$ as a function of $\mathrm{T}_{90}[3]$. 
Results shown in Fig. 2 support the choice of $\mathrm{T}_{90}=0.1 \mathrm{~s}$ to discriminate VSGRBs from SGRBs and show that the incompatibility with isotropic distribution is seen only for GRBs with $\mathrm{T}_{90}<$ $0.1 \mathrm{~s}$. We observe the total excess of $15.71 \pm 4.52$ bursts in this region.

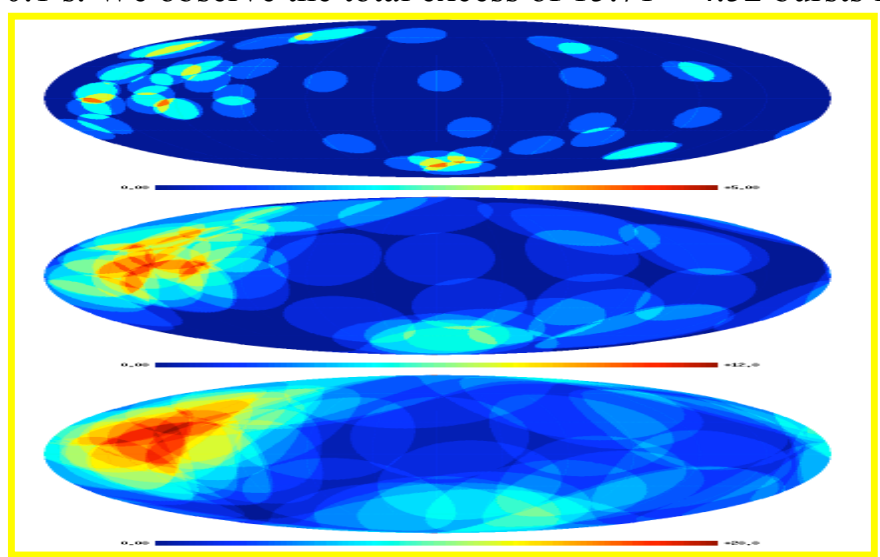

Fig. 3. Angular distribution of the BATSE VSGRB events in Galactic Coordinates within $10^{\circ}$ (top), $25^{\circ}$ (middle), and $40^{\circ}$ (bottom), radius cone around each event [4].

The detailed first four factorial moments analysis gives as a result the probability $<3 \cdot 10^{-5}$ for the chance of such fluctuation from uniform distribution [4]. This is in agreement with our earlier simple estimation: $7 \cdot 10^{-5}$ [3]. It means the effect itself is on about $4 \sigma$ level.

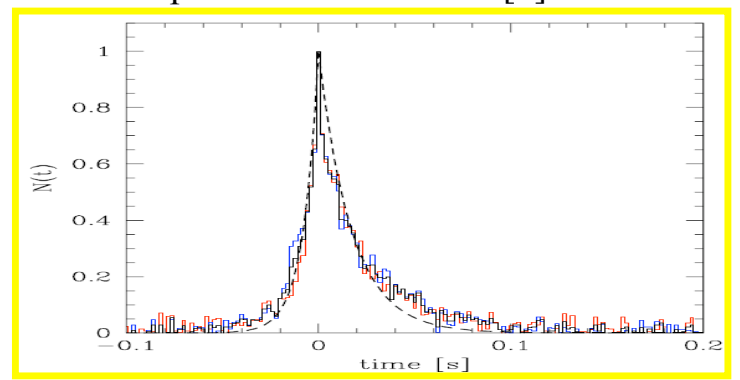

Fig. 4. Composite VSGRB time profile for BATSE; Dashed line - exponential fit [5].

The exponential fit was used twice (separately for rise and for fall). The resulting composite profile is shown in Fig. 4. It shows significant asymmetry with timescale: $0.00079 \mathrm{~s}$ for exponential rise and $0.0171 \mathrm{~s}$ for exponential decay time. If we use the evaporation PBH model, the fit to the rising part of the profile is good.

\section{References}

[1] D.B. Cline, C. Matthey and S. Otwinowski, Non-Isotropic Angular Distribution for Very ShortTime Gamma-Ray Bursts, UCLA-APH-0124-04-00[astro-ph/0105059].

[2] D.B. Cline et al, Evidence for Primordial Black Hole Final Evaporation: Swift, BATSE and KONUS and Comparisons of VSGRBs and Observations of VSB That Have PBH Time Signatures, astro$\mathrm{ph} / 0908.1352$.

[3] D.B. Cline et al, Study of Very Short GRB: New Results from BATSE and KONUS, ApJ. 633, L73 (2005) [astro-ph/0510309].

[4] L.W. Piotrowski et al, Angular Anisotropy of Very Short Gamma Ray Bursts, http://www.lip.pt/events/2006/ecrs/proc/ecrs06-s5-10.pdf.

[5] B.Czerny at al., Observational constraints on the nature of very short gamma-ray bursts, New Astronomy in press, [arXiv:1006.1470]. 